\title{
Mie's Theories of Matter and Gravitation
}

\author{
Christopher Smeenk and Christopher Martin
}

July 18, 2005

Unifying physics by describing a variety of interactions - or even all interactions - within a common framework has long been an alluring goal for physicists. One of the most ambitious attempts at unification was made in the 1910s by Gustav Mie. Mie aimed to derive electromagnetism, gravitation, and aspects of the emerging quantum theory from a single variational principle and a well-chosen Lagrangian. Mie's main innovation was to consider nonlinear field equations to allow for stable particle-like solutions (now called solitons), and he clarified the use of variational principles in the context of special relativity. The following brief introduction to Mie's work has three main objectives. ${ }^{1}$ The first is to explain how Mie's project fit into the contemporary development of the electromagnetic world view. Part of Mie's project was to develop a relativistic theory of gravitation as a consequence of his generalized electromagnetic theory, and our second goal is to briefly assess this work, which reflects the conceptual resources available for developing a new account of gravitation by analogy with electromagnetism. Finally, Mie was a vocal critic of other approaches to the problem of gravitation. Mie's criticisms of Einstein, in particular, bring out the subtelty and novelty of the ideas that Einstein used to guide his development of general relativity.

In September of 1913 Einstein presented a lecture on the current status of the problem of gravitation at the 85th Naturforscherversammlung. Einstein's lecture and the ensuing heated discussion, both published later that year in the Physikalische Zeitschrift, reflect the options available for those who took on the task of developing a new theory of gravitation. The

\footnotetext{
${ }^{1}$ Our study of Mie has been aided considerably by several recent discussions of his work, including Kohl (2002), Corry (1999), and Vizgin (1994, 26-38). Born (1914), included in this volume, gives an insightful, influential reformulation of Mie's framework, and Pauli (1921, §64, 188-192 in the English translation) and Weyl (1918, §25, 206-217 (§26) in the English translation of the fourth edition) both give clear contemporary reviews.
} 
conflict between Newtonian gravitational theory and special relativity provided a strong motivation for developing a new gravitational theory, but it was not clear whether a fairly straightforward modification of Newton's theory based on classical field theory would lead to a successful replacement. Einstein clearly aimed to convince his audience that success would require the more radical step of extending the principle of relativity. For Einstein the development of a new gravitational theory was intricately connected with foundational problems in classical mechanics, and in the Vienna lecture he motivated the need to extend the principle of relativity with an appeal to Mach's analysis of inertia. According to Einstein Mach had accurately identified an "epistemological defect" in classical mechanics, namely the introduction of a distinction between inertial and non-inertial reference frames without an appropriate observational basis. ${ }^{2}$ The special theory of relativity had replaced Galilean transformations between reference frames with Lorentz transformations, but the principle of relativity still did not apply to accelerated motion. Extending the principle of relativity to accelerated motion depended on an idea Einstein later called "the best idea of my life," the principle of equivalence. This idea received many different formulations over the years, but in 1913 Einstein gave one version of this principle as a postulate: his second postulate requires the exact equality of inertial and gravitational mass. He further argued that this equality undermines the ability to observationally distinguish between a state of uniform acceleration and the presence of a gravitational field. The principle of equivalence gave Einstein a valuable link between acceleration and gravitation, tying together the problem of gravitation and the problem of extending the principle of relativity. At the time of the Vienna lecture Einstein was in the midst of an ongoing struggle to clarify the connections among Mach's insight, a generalized principle of relavity, and the formal requirement of general covariance, a struggle that would continue for several more years. Although he also drew heavily on classical field theory in his work, he was convinced that this cluster of ideas would provide the key to a new theory of gravitation.

Gustav Mie's approach to the problem of gravitation stands in sharp contrast to Einstein's. In the discussion following the Vienna lecture, Mie pointedly criticized Einstein's requirement of general covariance and complained that Einstein had overlooked other approaches to gravitation, including his

\footnotetext{
${ }^{2}$ Einstein discusses these issues in $\S 4$ and $\S 9$ of the Vienna lecture, as well as in part II of Einstein (1914), both included in this volume. For a thorough discussion of the role of Machian ideas in Einstein's discovery of general relativity, see Renn (this volume).
} 
own work and that of Max Abraham. ${ }^{3}$ Mie commented that Einstein might have missed his theory of gravitation since it was "tucked away in a work on a comprehensive theory of matter" (CPAE 5, Doc. 18, 1262). This remark aptly characterizes where Mie placed the problem of gravitation conceptually; in Mie's approach the problem of gravitation would be solved as a byproduct of an extension of classical field theory. The problem of gravitation was one of the issues, among many, that a "comprehensive theory of matter" would resolve. The pressing issue for Mie was to develop a unified field theory that would succeed where earlier attempts at a reduction of mechanics to electromagnetic theory had failed. By way of contrast with Einstein, Mie's project did not lead out of special relativity, and Mie was not convinced by Einstein's attempt to link issues in the foundations of mechanics to the problem of gravitation. In Vienna, Einstein justified his sin of omission by pointing out that Mie's theory violated one of his starting assumptions, namely the principle of equivalence. But this clearly did not sway Mie, who expressed doubts that the principle could serve as the basis for a theory and whether it even held in Einstein's own "Entwurf" theory. ${ }^{4}$ Mie was also a forceful critic of Einstein's search for a generalized principle of relativity. In the discussion following the Vienna talk and in subsequent articles (Mie 1914 and 1915, included in this volume), Mie argued that Einstein had failed to establish a clear link between a principle of general relativity and accelerated motion and questioned the physical content of the principle. Mie had put his finger on the ambiguity of Einstein's guiding principles and the slippage between these ideas and the formal requirement of general covariance. More generally, Mie's criticisms illustrate that Einstein's idiosyncratic path to developing a new gravitational theory seemed to lead into the wilderness in 1913, and Einstein had not provided entirely convincing reasons to abandon a more conservative path toward a new theory.

Mie's comprehensive theory of matter was presented in a series of three ambitious papers in 1912-13. Mie was eleven years older than Einstein and had held a position as a theoretical physicist in Griefswald since 1902. He was well known for work in applied optics and electromagnetism, including an insightful treatment of the scattering of electromagnetic radiation by

\footnotetext{
${ }^{3}$ In the published version of the lecture Einstein does briefly mention Abraham's theory only to remark that it fails to satisfy his third postulate, namely the requirement of Lorentz covariance. Mie later noted (Mie 1914, note 13 on 175) that the reference to Abraham was only added in the published version of the lecture.

${ }^{4}$ In the discussion, Mie announced that he would soon publish a proof that equality does not hold in the "Entwurf" theory, which appeared in $\S 3$ of Mie (1914).
} 
spherical particles (Mie 1908) and a widely used textbook (Mie 1910). Mie's textbook endorsed the electromagnetic worldview prominently advocated in the previous decade by Wilhelm Wien and Max Abraham. This worldview amounted to the claim that electromagnetic theory had replaced mechanics as the foundation of physical theory, and Mie characterized electromagnetic theory as "aether physics." Mie emphasized the appeal of reducing physics to a simple set of equations governing the state of the aether and its dynamical evolution, and conceiving of elementary particles as stable "knots" in the aether rather than independent entities (Mie 1912a, 512-13). The aim of the trilogy on matter theory was to develop a unified theory able to account for the existence and properties of electrons (as well as atoms or molecules), explain recent observations of atomic spectra, and yield field equations for gravitation. Although Mie ultimately failed to achieve these grand goals, the approach and formalism he developed influenced later work in unified field theory.

Mie's program differed in important ways from electron theories from the previous decade. ${ }^{5}$ The main obstacle to earlier attempts to realize the electromagnetic world view was the difficulty of explaining the nature and structure of the electron itself in purely electromagnetic terms. Electron theory was an active research area in the first decade of the $20^{\text {th }}$ century, drawing the attention of many of the best physicists of that generation, such as Lorentz, Abraham, and Sommerfeld. By the time of Mie's work the aim of determining the internal structure of the electron, treated as an extended particle with a definite shape and charge distribution, had been largely abandoned and interest in electron theory had begun to wane. With the advent of special relativity came the realization that the velocity dependence of the electron's mass, a quantity that had been touted as a sensitive experimental test of the internal structure of the electron, was instead a direct consequence of the principle of relativity (Pauli 1921, 185; Pais 1972). ${ }^{6}$ Developments in electron theory also threatened the goal of replacing Newtonian mechanics with electromagnetism. Poincaré (1906) proved that an electron treated as a distribution of charge over a spherical shell is not a stable configuration if only the electromagnetic forces are included - the re-

\footnotetext{
${ }^{5}$ Here we draw primarily on Janssen and Mecklenburg (2005)'s insightful analysis of the transition from electron theory to relativistic electodynamics; see also the essays collected in Buchwald and Warwick (2001).

${ }^{6}$ Lorentz put the point as follows in 1922, "the formula for momentum is a general consequence of the principle of relativity, and a verification of that formula is a verification of the principle and tells us nothing about the nature of mass or of the structure of the electron," quoted in Janssen and Mecklenburg (2005).
} 
pulsive Coulomb forces would cause it to break apart. Thus it was necessary to introduce the so-called "Poincaré stress," an attractive force needed to maintain the stability of the electron.

One way of responding to these results was to temper the reductive ambitions of the electromagnetic world view, and to follow Lorentz in admitting charged particles or non-electromagnetic forces as basic elements of the theory. Mie took a different route, and chose instead to alter the field equations of electromagnetism so that there are solutions corresponding to stable particles. A successful theory along these lines would describe the fundamental particles as stable solutions to a set of field equations (with laws of motion derived directly from the field equations) without introducing particles as independent entities, and in this sense reduce mechanics to (generalized) electrodynamics. In effect, Mie treated Maxwell's equations as a weak-field limit of more general field equations. In order to allow for stable charge configurations such as an electron Mie considered non-linear field equations. The fundamental desideratum for the theory was to find generalized field equations that admitted stable solutions representing elementary particles and also reduced to Maxwell's equations in an appropriate limit for regions far from the particles. ${ }^{7}$ Mie further aimed to show that gravitation would naturally emerge as a consequence of the generalized field equations.

The key to Mie's theory was the "world function" (Hamiltonian), which he used to derive the field equations via Hamilton's principle. Maxwell's field equations in empty space follow from a Lagrangian $\Phi_{E M}=-\frac{1}{4} F_{\mu \nu} F^{\mu \nu}$, where $F_{\mu \nu}$ is the Maxwell tensor and the repeated indices (with $\mu, \nu=1 \ldots 4$ ) are summed over. Mie's program was to find the terms added to $\Phi_{E M}$ that would yield the desired generalized field equations. Mie introduced two fundamental assumptions regarding $\Phi$ at the outset of the Grundlagen. First, electrons and other charged particles should be regarded as "states of the aether" rather than independent entities. Mie insisted that the states of the aether should suffice for a complete physical description of matter, although he admitted that failure of his program might force one to enlarge the allowed fundamental variables. To enforce the first assumption Mie required that the world function depends only on the field variables (including the electric charge density, the convection current, the magnetic field strength,

\footnotetext{
${ }^{7}$ Mie was not the first to consider this way of extending classical electromagnetism. Prior to Mie's work Einstein considered replacing Maxwell's field equations with nonlinear, inhomogeneous, and/or higher order equations, as reflected in correspondence with Lorentz and Besso in 1908-1910 (see McCormach (1970) and Vizgin (1994), 19-26). Einstein, however, was much more keenly aware than Mie of the deep challenges posed by the quantum structure of radiation.
} 
and the electric displacement). As Born emphasized (1914, 32), this ruled out treating charged particles with trajectories given by independent equations of motion as the source of the field, since including a coupling to a background current in the Lagrangian (i.e., adding a term proportional to $J^{\mu} \phi_{\mu}$ ) would explicitly introduce dependence on spacetime coordinates. The second assumption was the validity of special relativity, with the consequence that $\Phi$ must be Lorentz covariant. The world function could only include functions of Lorentz invariant terms constructed from $F_{\mu \nu}$ and $\phi_{\mu}$, the four-vector potential. ${ }^{8}$ Mie argued that functions of only two of these invariants, namely $F_{\mu \nu} F^{\mu \nu}$ and $\phi_{\nu} \phi^{\nu}$ should appear in $\Phi$. For the general field equations to reduce to Maxwell's equations, the $\phi_{\nu} \phi^{\nu}$ term could have non-zero values only in regions occupied by particles.

Mie failed to flesh out his formalism with a specific world function satifying these constraints that led to a reasonable physical theory. Instead he was limited to illustrating his approach with simple examples, such as a Lagrangian $\Phi=-\frac{1}{4} F_{\mu \nu} F^{\mu \nu}+\alpha\left(\phi_{\nu} \phi^{\nu}\right)^{3}$, where $\alpha$ is an arbitrary constant. Solutions to the field equations that follow from this Lagrangian could be taken to represent elementary particles, and Mie calculated the charge and mass of the particles. However, these solutions had a number of undesirable features. The arbitrary coefficient appearing in the Lagrangian implied that these solutions placed no constraints on the charge and mass of the "particles," rather than leading to the distinctive values of charge and mass for known particles such as the electron. Mie was further forced to admit (1912b, 38) that his simple world function did not lead to reasonable solutions for interacting charged particles; instead the solutions described a world that eventually separated into two lumps of opposite charge moving away from each other. The simple world functions considered by Mie were not viable candidates for a comprehensive description of matter, but he clearly hoped that these problems could be blamed on his lack of ingenuity rather than on his formal framework. However, Pauli $(1921,192)$ highlighted a problem that went deeper than the failure to find a suitable world-function. Mie's world function and the resulting equations of motion both include functions

\footnotetext{
${ }^{8}$ Since $F_{\mu \nu}=\frac{\partial \phi_{\nu}}{\partial x_{\mu}}-\frac{\partial \phi_{\mu}}{\partial x_{\nu}}$, the Lagrangian depends on $\phi_{\nu}$ and its first derivatives. The list of invariants included the following quantities:

$$
\frac{1}{2} F_{\mu \nu} F^{\mu \nu} ; \quad \phi_{\nu} \phi^{\nu} ; \quad F_{\mu \nu} \phi^{\nu} F^{\mu \rho} \phi_{\rho} ; \quad\left(F_{\mu \nu} \phi_{\rho}+F_{\nu \rho} \phi_{\mu}+F_{\rho \mu} \phi_{\nu}\right)^{2}
$$

One invariant was missing from Mie's original list, as Pauli (1921) noted: the quantity $\frac{1}{4} F_{\mu \nu}^{*} F^{\mu \nu}$, where $F_{\mu \nu}^{*}$ is the dual of $F_{\mu \nu}$, is an invariant of the restricted Lorentz group, and its square is an invariant of the full Lorentz group.
} 
of $\phi_{\nu}$. As a result, a stable solution with some value of $\phi$ is in general not also a solution for $\phi+$ constant, and the world function also fails to be gauge invariant. ${ }^{9}$

Mie hoped that the appropriate world function (supposing one could be found) would incorporate gravity without needing to put it in by hand. At the outset of the Grundlagen Mie announced his goal of deriving gravity from his matter theory without introducing new dynamical variables and sketched a fanciful picture according to which gravity was a consequence of a cohesive shell or atmosphere binding particles together within an atom (Mie 1912a, 512-514). Mie's description of his project may have raised hopes that the third paper would introduce a truly novel approach to gravitation based on non-linear electrodynamics. But like his other grand goals, this one also eluded Mie's grasp.

Mie's gravitational theory has a great deal in common with competing theories due to Abraham and Nordström. Like Nordström, Mie retained an invariant speed of light and upheld the strict validity of the principle of relativity. This sets his approach apart from Abraham's work; Abraham renounced the constancy of the speed of light and retained the validity of the principle of relativity, restricted to infinitesimal space-time regions, in his first theory, and renounced the principle of relativity all together in his second theory. ${ }^{10}$ But like Abraham and Nordström, Mie treated both the source of the gravitational field and the gravitational potential to be fourdimensional (Lorentz) scalars, and these were introduced as independent quantities in the world function with no connection to the electromagnetic field. The source of the gravitational field, $h$, the density of gravitationalmass in Mie's theory, is identical to the Hamiltonian density. It is then a short step to derive field equations for the gravitational field appealing to Hamilton's principle. As Mie emphasized, the resulting field equations would be identical to those given by Abraham (1912) except for the introduction of another variable in the world function. ${ }^{11}$ By analogy with his matter theory, Mie introduced an extensive quantity (the excitation of the gravitational field, analogous to electric displacement) conjugate to the gravitational field

\footnotetext{
${ }^{9}$ Mie recognized this problem and argued that the resulting dependence on the absolute value of the potential would not lead to conflicts with experimental results (Mie 1912b, 24; Mie 1913, 62). Born and Infeld (1934) revived Mie's idea of using a more general Lagrangian, but they excluded additional terms that depended on $\phi_{\nu}$ to preserve gauge invariance.

${ }^{10}$ See Renn (this volume) and Norton (this volume) for discussions of Abraham's and Nordström's theories, respectively.

${ }^{11}$ See Mie (1913, 28-29) and the discussion following the Vienna lecture (CPAE 5, Doc. 18).
} 
strength, and argued that the two are identical in an "ideal vacuum" but have an unspecified functional relation in regions occupied by matter.

Mie's failure to achieve a substantial unification illustrates the obstacles to treating gravitation by analogy with electromagnetism. Mie (1915) clearly explained the necessity of introducing the gravitational potential as a dynamical variable in order to resolve the negative energy problem, the most important disanalogy. This problem arises if energy is attributed to the gravitational field itself (as with the electromagnetic field), since the gravitational field strength of, for example, two gravitating masses increases as two masses approach each other, releasing energy in the form of work extracted from the system. One way to save energy conservation in light of this feature of gravitation was to attribute negative energy to the gravitational field, as is suggested by treating Newtonian gravitation in close formal analogy to electrostatics. However, a field with negative energy cannot maintain a stable equilibrium since any small perturbation of the field would in general grow without limit. Following Abraham, Mie argued that the way out of this dilemma was instead to include the gravitational potential in the world-function. With this, the internal energy of two approaching masses can be shown to decrease with the decrease of the gravitational potential, thereby compensating for the increase in the field energy.

Including the gravitational potential as a dynamical variable has the consequence that, unlike in electromagnetism, the equations governing physical phenomena depend upon the absolute value of the potential rather than on just potential differences. However, no such dependence had been empirically detected. It remains, moreover, to specify exactly how the field energy depends on the gravitational potential. As Mie (1915) noted, different choices for this dependence correspond to different gravitational theories. Given the lack of empirical guidance to settle the issue, Mie argued in favor of introducing a principle that would dictate this dependence rather than making what he regarded as arbitrary assumptions. Mie hoped to reconcile his theory's explicit dependence on the absolute value of the gravitational potential with the failure to experimentally detect any such dependence via the theorem (later called a principle) of the relativity of the gravitational potential (discussed below). The principle plays a central role in the development of Mie's theory, and in elucidating this idea Mie drew a sharp contrast between his approach and Einstein's insistence on generalizing the principle of relativity.

Mie (1915) formulated the principle of the relativity of the gravitational potential as follows: 
In two regions of different gravitational potential exactly the same processes can run according to exactly the same laws if one only thinks of the units of measurement as changing in a suitable way with the value of the gravitational potential. (Mie $1915,257)$

In order to understand the content of this principle, it is perhaps helpful to consider that the principle is equivalent to the requirement that the world function $\Phi$ be a homogenous function of the dynamical variables, including the gravitational potential (Mie $(1915,258)$ ). From this it immediately follows that in regions of constant gravitational potential, one can transform the potential away, or into any other constant potential, through a rescaling of the remaining dynamical variables and, in general, the space time coordinates. Thus, for an observer using correspondingly rescaled measuring units to measure the dynamical variables, the gravitational potential will be undetectable. Thinking of Mie's principle of relativity of the gravitational potential along these lines as an invariance of the theory under rescaling, we see the gravitational potential and rescalings in Mie's theory as anologous, respectively, to the the metric tensor and general linear transformations in Einstein's tensor theory. ${ }^{12}$ Simply put, Mie introduces the gravitational potential into the world function to solve the negative energy problem, and introduces an invariance principle, the principle of the relativity of the gravitational potential, to remove any dependence of physical laws on the potential.

By contrast with Einstein, Mie's introduction of this invariance principle for the gravitational field had no connection with foundational problems in mechanics or with extending the principle of relativity. Mie was clearly quite skeptical of the heuristic value of Einstein's guiding ideas. In the discussion following the Vienna lecture, Mie pointedly criticized the idea of extending the principle of relativity to arbitrary states of motion. Mie pressed Einstein to clarify what would be gained by treating a complicated non-uniform motion, such as a bumpy train ride, as physically equivalent to the gravitational field produced by some array of fictitious planets (CPAE 5, Doc. 18). The underlying problem stemmed from Einstein's failure to distinguish between two claims. In the familiar cases of relativity of uniform motion, the two systems in relative motion are entirely physically equivalent. But Einstein's

\footnotetext{
${ }^{12}$ Our understanding here was guided by CPAE 8, Doc. 346, fn. 3. Note that in his earlier work, Mie $(1912,61)$ refers to this as the theorem of the relativity of the gravitational potential. Even at this early juncture, though, Mie is quick to elevate this theorem, immediately dubbing it a principle.
} 
extension of relativity to non-uniform motion involves a very different claim; as he would later clarify, what is relative in the case of non-uniform motion is how the metric field is split into inertial and gravitational components. This does not, however, imply that two observers in non-uniform motion with respect to each other are physically equivalent. In 1913 Einstein did not answer Mie by drawing this distinction; instead, he replied that his theory did not satisfy an entirely general principle of relativity due to a restriction on allowed coordinate transformations (needed, Einstein thought, to insure energy-momentum conservation). Mie (1914) further argued that since the "Entwurf" theory admits only general linear transformations, it does not realize a general principle of relativity, but in fact satisfies precisely Mie's principle of the relativity of the gravitational potential.

Einstein's equivalence principle was also a target of Mie's criticisms. This is not surprising, since Mie's commitment to retaining the framework of special relativity implied that in his theory inertial and gravitational mass would not be exactly equal. Mie $(1915, \S \S 5 \& 6)$ calculated the effect of the thermal motions of the constituents of bodies on this ratio, and argued that departures from 1 would be well within experimental bounds. Exact equivalence could be had at the price of various auxiliary assumptions, according to Mie, but he did not see the need for such extra assumptions, given that his theory fit experimental constraints. He further claimed that Einstein's theory can only guarantee exact equivalence by making inconsistent assumptions (Mie 1914, 176). This attitude toward the equivalence principle marks another contrast with Einstein, who took the "unity of essence" of inertia and gravitation to be one of the central foundational insights to be respected by his new theory.

In summary, Mie's work illustrates the potential and limitations of approaching the problem of gravitation within the framework of relativistic field theory. Mie's main innovation in the Grundlagen was to consider nonlinear field equations, which opened up the possibility of reducing physics to an electromagnetic matter theory. The appeal of this idea has to be balanced against the theory's glaring deficiency, namely the failure to find a particular world function describing even a simple physical system such as two interacting particles. To paraphrase Einstein, although Mie's theory provided a fine formal framework, it was not clear how to fill it with physical content. ${ }^{13}$ Even those sympathetic to Mie's program had to ad-

\footnotetext{
${ }^{13}$ In a 1922 letter to Weyl regarding Eddington's later attempt at a unified field theory, quoted in Vizgin $(1994,37)$, Einstein commented that "I find the Eddington argument to have this in common with Mie's theory: it is a fine frame, but one cannot see how it can be filled"; see also his negative assessments of Mie's theory (directly or as it was used by
} 
mit doubts that this innovation would lead to a successful matter theory, especially given the recent discoveries of quantum phenomena. But whatever the prospects for matter theory based on generalized electrodynamics, Mie's innovations in the Grundlagen turned out to provide few insights for developing a gravitational theory. His own gravitational theory shared the insights and limitations of other Lorentz-covariant theories of gravitation. In terms of the further development of gravitational theories, Mie's indirect influence on David Hilbert is more significant than his own theory. This influence was mediated by Born (1914)'s clear reformulation of Mie's theory, which showed how Mie's theory fit into the more general framework of (four-dimensional) continuum mechanics as a special case. Mie's project of unification and his mathematical framework, as refined by Born, shaped Hilbert's distinctive path to a new gravitational theory, as Renn and Stachel (this volume) explore in great detail. ${ }^{14}$ However, Hilbert differed from Mie sharply with regard to the status of special relativity. Mie was a persistent critic of Einstein's move to a metric theory of gravitation and saw no reason to leave the framework of special relativity. Hilbert, on the other hand, took Einstein's "Entwurf" theory as one of his starting points, and his synthesis of Mie's matter theory with Einstein's gravitational theory involved replacing the fixed Minkowski metric of special relativity with Einstein's metric tensor. The fertility of Mie's matter theory for Hilbert depended upon setting aside Mie's criticisms of Einstein's move away from special relativity.

\section{References}

Born, Max. 1914. "Der Impuls-Energie-Satz in der Elektrodynamik von Gustav Mie." Königliche Gesellschaft der Wissenschaften zu Göttingen. Nachrichten (1914): 2336.

Buchwald, Jed Z., and Andrew Warwick (eds.). 2001. Histories of the Electron. The Birth of Microphysics. Cambridge: The MIT Press.

CPAE 4: Martin J. Klein, A. J. Kox, Jürgen Renn, and Robert Schulmann (eds.). 1995. The Collected Papers of Albert Einstein. Vol. 4. The Swiss Years: Writings, 19121914. Princeton: Princeton University Press.

CPAE 6: A. J. Kox, Martin J. Klein, and Robert Schulmann (eds.). 1996. The Collected Papers of Albert Einstein. Vol. 6. The Berlin Years: Writings, 19141917. Princeton: Princeton University Press.

Hilbert) in letters to Freundlich (CPAE 5, Doc. 468), Ehrenfest (CPAE 8, Doc. 220) and Weyl (CPAE 8, Doc. 278). Weyl gave a similar assessment of Mie's theory; see $\S 25$ of Weyl (1918); pp. 214-216 of the English translation.

${ }^{14}$ See also Sauer (1999) and Corry (1999) for assessments of Hilbert's project and the influence of Mie's theory. 
CPAE 6E: The Collected Papers of Albert Einstein. Vol. 6. The Berlin Years: Writings, 1914191\%. English edition translated by Alfred Engel, consultant Engelbert Schucking. Princeton: Princeton University Press, 1996.

CPAE 8: Robert Schulmann, A. J. Kox, Michel Janssen, and József Illy (eds.). 1998. The Collected Papers of Albert Einstein. Vol. 8. The Berlin Years: Correspondence, 19141918. Princeton: Princeton University Press.

CPAE 8E: The Collected Papers of Albert Einstein. Vol. 8. The Berlin Years: Correspondence, 19141918. English edition translated by Ann M. Hentschel, consultant Klaus Hentschel. Princeton: Princeton University Press, 1998.

Corry, Leo. 1999. From Mie's Electromagnetic Theory of Matter to Hilbert's Unified Foundations of Physics. Studies in History and Philosophy of Modern Physics 30 B (2):159183.

Einstein, Albert. 1913. "Zum gegenwärtigen Stande des Gravitationsproblems." Physikalische Zeitschrift 14 25: 12491262.

Einstein 1914

Janssen, Michel and Mecklenburg, Matthew. Forthcoming. "The Transition from Classical to Relativistic Mechanics: Electromagnetic Models of the Electron." To appear in a volume edited by Jesper Lützen based on the proceedings of the conference, The interaction between Mathematics, Physics and Philosophy from 1850 to 1940, Copenhagen, September 2628, 2002. Earlier version available as Max Planck Institute for the History of Science Preprint 277.

Kohl, Gunter. 2002. "Relativität in der Schwebe: Die Rolle von Gustav Mie." Max Planck Institute for the History of Science Preprint 209.

McCormach, 1970

Pais, Abraham, 1972. "The Early History of the Theory of the Electron: 18971947." In Aspects of Quantum Theory. Edited by Abdus Salam and Eugene P. Wigner. Cambridge: Cambridge University Press, 7993.

Pauli, Wolfgang. 1921. Relativitätstheorie. In Encyklopädie der mathematischen Wissenschaften, mit Einschluss ihrer Anwendungen, edited by Arnold Sommerfeld. Leipzig: B. G. Teubner, 539775.

. 1958. Theory of Relativity. Translated by G. Field. London: Pergamon. Renn, Jürgen. 1994. The Third Way to General Relativity. Included in this volume.

Sauer, Tilman. 1999. The Relativity of Discovery: Hilberts First Note on the Foundations of Physics. Archive for History of Exact Sciences 53: 529575 . 
Vizgin, Vladimir. 1994. Unified Field Theories in the First Third of the 20th Century. Translated by Barbour, Julian B. Edited by E. Hiebert and H. Wussing. Vol. 13, Science Networks, Historical Studies. Basel, Boston, Berlin: Birkhäuser.

Weyl, Hermann. 1918. Raum-Zeit-Materie. Vorlesungen über allgemeine Relativitätstheorie. 1st ed. Berlin: Julius Springer. 